\title{
Sparse massive MIMO-OFDM channel estimation based on compressed sensing over frequency offset environment
}

\author{
Guang Li*, Tianyun Li, Mankun Xu, Xiong Zha and Yunfei Xie
}

\begin{abstract}
In massive MIMO-OFDM systems, channel estimation is a significant module which can be utilized to eliminate multipath interference. However, in realistic communication systems, carrier frequency offset (CFO), which often exists in receive end, will deteriorate the performance of channel estimation. One of the effective solutions is to compensate CFO via the help of pseudo-noise (PN) sequence. At the beginning of this paper, to reduce system complexity and correctly compensate CFO, we propose an improved OFDM frame structure. Subsequently, we theoretically analyze the catastrophic influence of CFO on conventional PN-sequence-based compressed sensing (CS) channel estimation scheme. As our solution, based on the improved OFDM frame structure, a novel massive MIMO-OFDM channel estimation method under CFO environment is proposed. It first estimates CFO by utilizing differential correlation algorithm. Thereby, the interference caused by CFO can be eliminated. Then, relying on the PN sequence, the partial common support (PCS) information of each channel is obtained. Finally, using the PCS information as a priori information, we improve the CS reconstruction scheme to estimate the accurate channel. The simulation result shows that the proposed scheme demonstrates better MSE and BER performance than other mentioned schemes. The major advantage of our scheme is its anti-CFO ability and independence to channel sparsity level. Therefore, the proposed scheme is meaningful for practical use.
\end{abstract}

Keywords: Channel estimation, Massive MIMO-OFDM, Carrier frequency offset, Partial common support, PN sequence, Compressed sensing

\section{Introduction}

\subsection{Background knowledge}

As an efficient technique for $5 \mathrm{G}$ (the 5th generation) wireless communication systems, massive multiple input multiple output orthogonal frequency division multiplexing (MIMO-OFDM) is widely applied by virtue of its excellent properties in data transmission rate and accuracy [1-3]. It is well known that precise channel estimation is a significant module to maintain the performance of MIMOOFDM [4, 5]. However, compared with orthogonal frequency division multiplexing (OFDM), accurate channel estimation is more challenging in massive MIMO-OFDM systems. Because for each receive antenna, there are many channels need to be estimated which is corresponding to all of the transmit antennas. In addition, the carrier

\footnotetext{
* Correspondence: 1226813282@qq.com

PLA Strategic Support Force Information Engineering University, Zhengzhou 450000, China
}

frequency of the receiver is difficult to be exactly same as that of the transmitter, leading to carrier frequency offset (CFO) at the receive end, which seriously reduces the accuracy of channel estimation [6]. Therefore, it is necessary to compensate $\mathrm{CFO}$ at the receive end before channel estimation.

In actual MIMO-OFDM communication systems, accurate channel estimation is obtained by the aid of training data. The frequency-domain pilot is the most common training data [7]. In the past, lots of research focusing on pilot-based channel estimation has been done. Some conventional channel estimation methods are proposed such as least square (LS) algorithm and minimum mean square error (MMSE) algorithm $[8,9]$. However, the performance of the LS is inadequate and greatly affected by noise. MMSE can achieve much better detection performance by utilizing the second order statistics of channels. However, it suffers from high computational complexity [10]. Moreover, 
the insertion of the pilot pattern has to satisfy Nyquist Sampling Theorem in both time domain and frequency domain, which leads to a reduction in spectrum utilization [11].

Studies have shown that wireless channels are usually sparse, especially for cellular massive MIMO-OFDM systems [12]. In other words, the tap coefficients of most paths in channel impulse response (CIR) can be regarded as zero. Moreover, for a given receive antenna, the channel delays of all transmit antenna can be considered to be consistent due to the small transmission base station array. In order to take advantage of the channel sparsity, we introduced the compressed sensing (CS) theory to estimate channel in MIMO-OFDM systems [13]. One of the advantages is that the pilots needed in CS-based channel estimation are much less than that of LS and MMSE [14]. Literature [15] uses orthogonal matching pursuit (OMP) and compressed sampling matching pursuit (CoSaMP) to achieve channel estimation of MIMO-OFDM systems. The main drawback of those schemes is that the channel sparsity level is regarded as a priori information in receive end [16]. Unfortunately, the channel sparsity level is unknown in the actual communication process, which limits their application in practice. Sparsity adaptive matching pursuit (SAMP) is a promising candidate scheme for many practical applications when the channel sparsity is unavailable [17]. It reconstructs channel information through stage by stage estimation of the sparsity level and the true support set of the target signals [18]. However, one of the shortcomings of SAMP is the fixed step size, which easily results in the contradiction between the convergence speed and the recovery accuracy of the algorithm $[19,20]$.

Recently, the CS channel estimation based on partial channel support (PCS) became a research hotspot. PCS mainly includes part of channel delay and path number. In [21], authors have proposed a CS-based channel estimation method for MIMO-OFDM by simultaneously exploiting the time-domain pseudo-noise ( $\mathrm{PN}$ ) sequence and frequency-domain pilots. It utilizes the autocorrelation of the PN sequence to acquire the PCS information. Then, the OFDM blocks are cyclically reconstructed based on the idea of overlap and add (OLA) algorithm. Finally, by exploiting the PCS as a priori information, the subspace pursuit (SP) algorithm is optimized and accurate CIR is reconstructed. To precisely remove the inter-block interference (IBI) between PN sequence and OFDM block in the OFDM block reconstruction, authors of [22] have proposed the dual-PN padded OFDM (DPN-OFDM) scheme, whereby the PN sequence is duplicated twice to make the second PN sequence immune from the IBI caused by the preceding OFDM block. Authors of [23] have obtained the channel estimation of DPN-OFDM system by using the method in [21]. However, neither [21] nor [23] has taken the effect of CFO into account. But the fact is that the autocorrelation performance of PN sequence decreases sharply over CFO environment [24]. Consequently, the PCS could not be obtained and the CSbased channel estimation is out of effect. In addition, due to the reconstruction of OFDM blocks, the scheme mentioned in [21] is complicated in implementation.

In order to eliminate the influence of CFO on PCS information acquisition, differential correlation algorithm based on PN sequence is discussed in the literature $[25,26]$, where $\mathrm{m}$-sequence is served as $\mathrm{PN}$ sequence. In [25], the differential delay is set to one, and an ambiguity protection module is used to alleviate the cross term effect of the multipath. In [26], the differential delay is set to a specific value $D$, so the frequency estimation range is limited. By applying the differential correlation algorithm twice with different $D$, more accurate frequency estimation can be achieved.

\subsection{Contributions}

In this paper, we discuss the channel estimation of massive MIMO-OFDM systems over frequency offset environment. The main contributions of the paper are summarized as follows:

1. Motivated by the frame structure of cyclic prefix OFDM (CP-OFDM) [27], we propose a novel OFDM frame structure. Its major benefit is that there is no need to cyclically reconstruct the OFDM block. Moreover, it enhances the CFO compensation performance.

2. We eliminate the influence of $\mathrm{CFO}$ on channel estimation via exploiting differential correlation algorithm to obtain the PCS information under CFO environment.

3. We improve the accuracy of CS reconstruction algorithm by utilizing the PCS information and appropriately setting the iteration termination threshold.

The remainder of this paper is organized as follows. In Section 2, the system model is presented. Section 3 introduces the proposed OFDM frame structure. Section 4 introduces the PCS information acquisition and the CFO effect on it. Section 5 illustrates the CFO compensation. Afterwards, the improved CS reconstruction algorithm is presented in Section 6. Section 7 gives the experimental results and comparison analysis. Finally, conclusions are given in Section 8.

Notions: For a given matrix $\mathrm{A}, \mathrm{A}^{\mathrm{T}}, \mathrm{A}^{-1}$, and $\|\mathrm{A}\|$ denote its transpose, inverse, and $l_{2}$ norm. $\left.\mathrm{A}\right|_{\lambda}$ is the $\lambda$ th column of A. $\mathrm{I}_{N}$ is an $N \times N$ identity matrix. $\mathbb{C}^{M \times N}$ represents the set of $M \times N$ matrices in the complex field. $\mathrm{CN}(\alpha, \beta)$ 
is a Gaussian random vector with mean $\alpha$ and covariance $\beta$.

\section{System model}

In this paper, we consider a $N_{t} \times N_{r}$ massive MIMOOFDM system which consists of $N_{t}$ transmit antennas and $N_{r}$ receive antennas. Due to the multipath property of wireless channel, the time-domain CIR from the $n_{t}$ th transmit antenna to the $n_{r}$ th receive antenna is given as

$$
h_{n_{t} n_{r}}(t)=\sum_{i=0}^{L-1} \alpha_{i} g\left(t-\tau_{i} T_{s}\right)
$$

where $\tau_{L-1}>\cdots>\tau_{0}$ represents the paths' delay, $\alpha_{i}$ is the tap coefficient of the $i$ th path, $g(\cdot)$ is the shaping pulse, and $T_{s}$ represents the sampling period [27]. For convenience of expression, we rewrite $h_{n_{t} n_{r}}(t)$ in a discrete form as

$$
\mathrm{h}_{n_{t} n_{r}}=\left[h_{n_{t} n_{r}}(0), h_{n_{t} n_{r}}(1), \ldots, h_{n_{t} n_{r}}(L-1)\right]^{T} \in \mathbb{C}^{L \times 1}
$$

Assuming that the number of subcarriers for each OFDM symbol is $N$, we denote $\mathrm{X}^{n_{t}}=\left(X_{1}^{n_{t}}, X_{2}^{n_{t}}, \ldots, X_{N}^{n_{t}}\right)^{T}$ $\in \mathbb{C}^{N \times 1}$ as the transmitted symbol of the $n_{t}$ th $\left(1 \leq n_{t} \leq N_{t}\right)$ transmit antenna. Since the pilot-based CS channel estimation is discussed in this work, we suppose that $N_{p}$ pilots are inserted at each OFDM symbol. The transmitted pilots of the $n_{t}$ th $\left(1 \leq n_{t} \leq N_{t}\right)$ transmit antenna can be expressed as $X_{P}^{n_{t}}=\left(X_{k_{1}}^{n_{t}}, X_{k_{2}}^{n_{t}}, \ldots, X_{k_{N_{P}}}^{n_{t}}\right)^{T} \in \mathbb{C}^{N_{P} \times 1}$, where [ $\left.k_{1}, k_{2}, \ldots, k_{N_{p}}\right]$ denotes the location of the pilot. The signal received at the $n_{r}$ th $\left(1 \leq n_{r} \leq N_{r}\right)$ receive antenna can be written as

$$
\begin{aligned}
\mathrm{Y}^{n_{r}} & =\sum_{n_{t}=1}^{N_{t}} \operatorname{diag}\left(\mathrm{X}^{n_{t}}\right) \mathrm{Fh}_{n_{t} n_{r}}+\mathrm{n}_{n_{r}} \\
& =\sum_{n_{t}=1}^{N_{t}} \operatorname{diag}\left(\mathrm{X}^{n_{t}}\right) \mathrm{H}_{n_{t} n_{r}}+\mathrm{n}_{n_{r}}
\end{aligned}
$$

where

$$
\mathrm{Y}^{n_{r}}=\left[Y_{1}^{n_{r}}, Y_{2}^{n_{r}}, \ldots, Y_{N}^{n_{r}}\right]^{T} \in \mathbb{C}^{N \times 1}
$$

and

$$
\mathrm{H}_{n_{t} n_{r}}=\left[H_{n_{t} n_{r}}(1), H_{n_{t} n_{r}}(2), \ldots, H_{n_{t} n_{r}}(N)\right]^{T} \in \mathbb{C}^{N \times 1}
$$

is the frequency domain CIR from the $n_{t}$ th transmit antenna to the $n_{r}$ th receive antenna, $\mathrm{F} \in \mathbb{C}^{N \times N}$ denotes an $N$-point normalized discrete Fourier transform (DFT) matrix with

$$
[\mathrm{F}]_{n, m}=1 / \sqrt{N} \exp (-j 2 \pi n m / N) \quad 0 \leq n \leq N-1,0 \leq m \leq L-1
$$

and $\mathrm{n}_{n_{r}} \sim C N\left(0, \sigma_{n}^{2}\right)$ is the additive noise vector [28].

\section{The proposed OFDM frame structure}

Figure 1a plots the existing OFDM frame structure [21], as shown, the PN sequence at a certain transmission antenna overlaps the others, which interferes the estimation of CFO between different transmit-receive antenna pairs. Moreover, as shown in Fig. 1b, the signal reconstruction in this OFDM frame structure is very complicated due to the overlap and add (OLA) algorithm. In addition, the inter-block interference (IBI) cannot be correctly obtained before channel estimation, so the reconstruction performance cannot be guaranteed.

To avoid the complex OFDM block reconstruction, a novel OFDM frame structure is proposed here which is shown in Fig. 2. The application background of this frame structure is the time-varying multipath wireless channel environment. More specifically, during one OFDM frame period, only the CIR tap coefficient is time-varying but the paths' delay stays unchanged.

Unlike the OFDM frame structure mentioned in [21, 28], as shown in Fig. 2a, the proposed frame structure, the PN sequence only exists in the front of the whole frame, and CP is inserted in front of each OFDM block. The major advantage is twofold. On the one hand, since the paths' delay stays unchanged in an OFDM frame duration, the PN sequence can be employed to precisely obtain the PCS information for each OFDM symbol; hence, the superior performance of CS-based channel estimation can be guaranteed. On the other hand, the inserted CP can be used to eliminate the interference between PN sequence and OFDM block, which is caused by channel multipath; thus, there is no need to reconstruct the OFDM blocks, which is shown in Fig. 2b.

For the purpose to estimate the channel between different transmit-receive antenna pairs, PN sequence inserted in different antennas should not be the same. The conventional approach is to insert a different $\mathrm{PN}$ sequence in the same position at different antennas [29]. However, under the multipath channel, the cross-correlation of different $\mathrm{PN}$ sequences in the receive end will affect the detection of the correlation peak. In the proposed OFDM frame structure, the PN sequence location at different antennas is orthogonal. More specifically, as shown in Fig. 2a, the front part of each OFDM frame is composed of one PN sequence and some zero sequences, and there is no overlap between the locations of different $\mathrm{PN}$ sequences. 


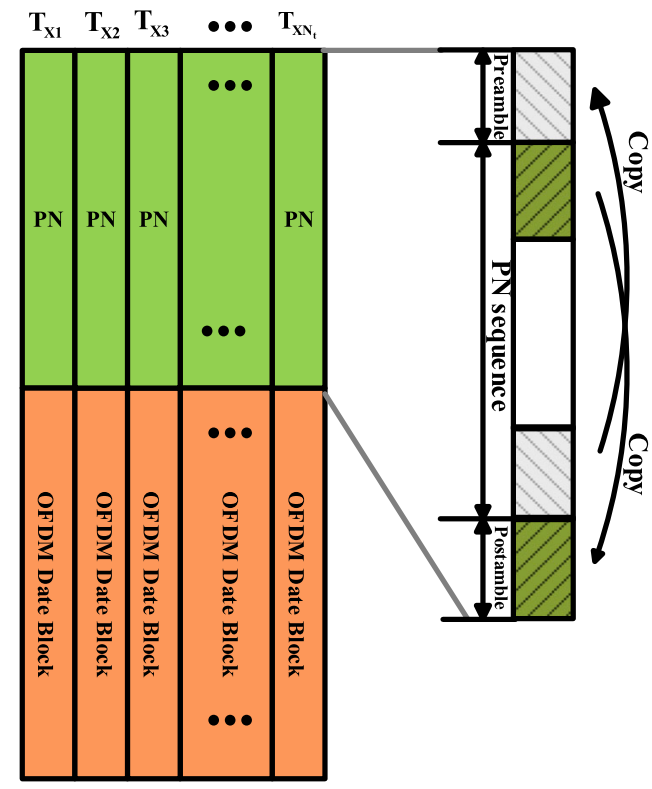

(a) frame structure

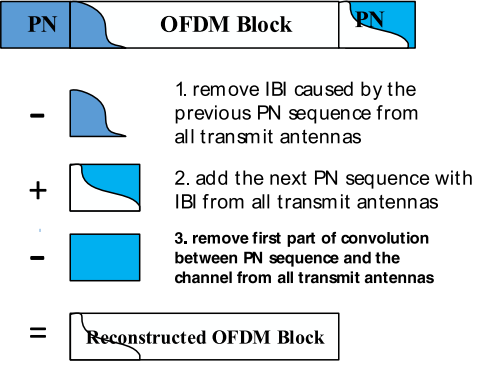

(b) OFDM block reconstruction

Fig. 1 The existing OFDM frame structure

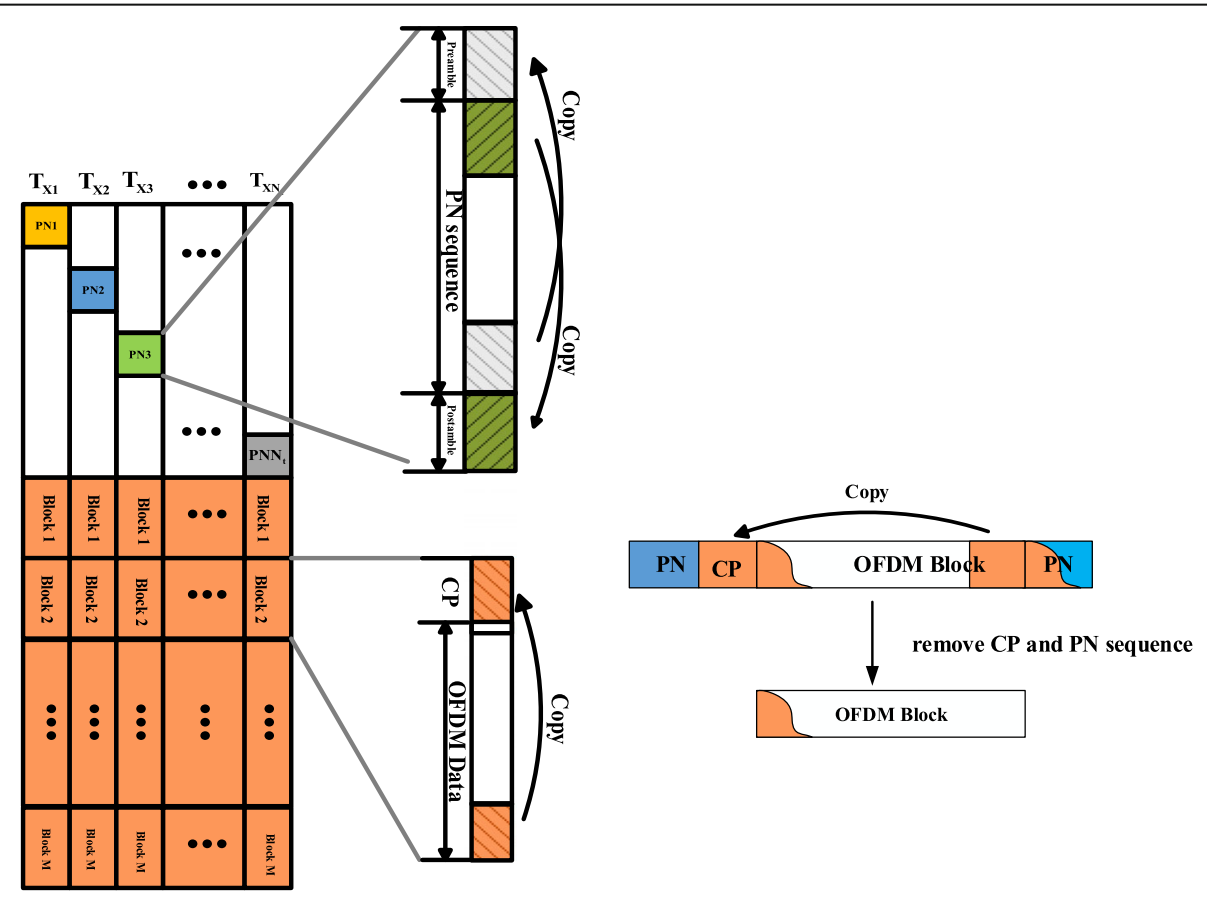

(a) frame structure

(b) OFDM block reconstruction

Fig. 2 The proposed OFDM frame structure 


\section{PCS information acquisition and the CFO effect on it}

The PCS information acquisition is based on the autocorrelation property of the PN sequence. For analysis purpose, the noise is neglected in this section. When there is the known PN sequence in the received signal $r(n)$, the cross-correlation between $r(n)$ and $c(n)$ can be expressed as

$$
\begin{aligned}
R_{r c}(s) & =\frac{1}{M} \sum_{n=0}^{M-1} c(n) \cdot r^{*}(n+s) \\
& =\left\{\begin{aligned}
1 & , \quad s=0 \\
-\frac{1}{M}, & \text { otherwise }
\end{aligned}\right.
\end{aligned}
$$

where $s$ is the shift in the sampling period [30]. Due to the excellent anti-noise performance of the PN sequence, the peak of $R_{r c}(s)$ will appear at the starting position of the PN sequence $(s=0)$. Now, we consider the effect of the multipath channel, and the received $\mathrm{PN}$ sequence which is the transmitted form $n_{t}$ th transmit antenna to $n_{r}$ th receive antenna can be rewritten as

$$
r(n)=\sum_{l=0}^{L-1} h_{n_{t} n_{r}}(l) c(n-l)
$$

and $R_{r c}(s)$ is given by

$$
\begin{aligned}
R_{r c}(s) & =\frac{1}{M} \sum_{n=0}^{M-1} c(n) \cdot \sum_{l=0}^{L-1} h_{n_{t} n_{r}}^{*}(l) c^{*}(n+s-l) \\
& =\frac{1}{M} \sum_{l=0}^{L-1} h_{n_{t} h_{r}}^{*}(l) \sum_{n=0}^{M-1} c(n) c^{*}(n+s-l) \\
& =\left\{\begin{array}{cc}
h_{n_{t} h_{r}}^{*}(s)+\varepsilon_{1} & , s \in[0, L-1] \\
\varepsilon_{2} & , \text { otherwise }
\end{array}\right.
\end{aligned}
$$

where

$$
\begin{aligned}
& \varepsilon_{1}=-\frac{1}{M} \sum_{l=0, l \neq s}^{L-1} h_{n_{t} n_{r}}^{*}(l) \\
& \varepsilon_{2}=-\frac{1}{M} \sum_{l=0}^{L-1} h_{n_{t} n_{r}}^{*}(l)
\end{aligned}
$$

From Eq. (9), in the case of the multipath channel, the $R_{r c}(s)$ is the superposition of the cross-correlation between multiple delay extended $\mathrm{PN}$ sequences and local PN sequence. Consequently, there are $L$ peaks in $R_{r c}(s)$. In $[21,28]$, the location of those peaks that exceed the threshold $\xi$ is extracted as PCS information.

$\mathrm{CFO}$ is common in the actual communication process and usually caused by the error between the local carrier frequency of the receiver and the real carrier frequency of the transmitter [31]. In general, OFDM systems are very sensitive to $\mathrm{CFO}$ as it destroys the orthogonality of subcarriers. Moreover, for the application of this paper, the CFO will introduce a time-dependent linear phase and distort the autocorrelation of the $\mathrm{PN}$ sequence. Assuming that the CFO is $f_{c}$ subcarrier spacing, the received $\mathrm{PN}$ sequence including $\mathrm{CFO}$ effect can be expressed as

$$
r(n)=\sum_{l=0}^{L-1} h_{n_{t} n_{r}}(l) c(n-l) e^{j 2 \pi(n-l) f_{c}}
$$

And the CFO-effected $R_{r c}(s)$ is given by

$$
\begin{aligned}
R_{r c}(s) & =\frac{1}{M} \sum_{l=0}^{L-1} h_{n_{t} n_{r}}^{*}(l) \sum_{n=0}^{M-1} c(n) c^{*}(n+s-l) e^{j 2 \pi(n+s-l) f_{c}} \\
& =\left\{\begin{array}{cc}
h_{n_{t} n_{r}}^{*}(s) \frac{1}{M} \sum_{n=0}^{M-1}|c(n)|^{2} e^{j 2 \pi n f_{c}}+\Delta \varepsilon_{1}, s \in[0, L-1] \\
\Delta \varepsilon_{2}, & \text { otherwise }
\end{array}\right. \\
& =\left\{\begin{array}{cc}
h_{n_{t} n_{r}}^{*}(s) e^{j \pi(M-1) f_{c}} \operatorname{sinc}\left(\pi M f_{c}\right)+ & \Delta \varepsilon_{1}, s \in[0, L-1] \\
\Delta \varepsilon_{2}, & \text { otherwise }
\end{array}\right.
\end{aligned}
$$

where $\Delta \varepsilon_{1}$ and $\Delta \varepsilon_{2}$ are the interference terms related to $\varepsilon_{1}$ and $\varepsilon_{2}$, respectively, and $\operatorname{sinc}(\cdot)$ is the abbreviation of Singer function. It can be observed from Eq. (12) that the amplitude of the correlation peaks is reduced in Singer function way. More seriously, our simulation result shows that the correlation peaks will disappear when CFO is large enough. In other words, the PCS information cannot be obtained precisely in this case.

\section{CFO compensation}

In this section, we will focus on the CFO compensation. Since PN sequence is exploited to obtain PCS information, to reduce computation and save frequency resource, here, we still use PN sequence to compensate CFO. The traditional PN-based CFO compensation scheme is coherent autocorrelation algorithm.

\subsection{Coherent autocorrelation algorithm}

After the frame synchronization is completed, the conjugated $\mathrm{PN}$ sequence generated by the receive 
$c^{*}(n)$ is multiplied by the received PN sequence $r(n)$ to get $z(n)$

$$
z(n)=r(n) c^{*}(n)=\sum_{l=0}^{L} h_{n_{t} n_{r}}(l)|c(n)|^{2} e^{j\left(2 \pi f_{c} n+\theta\right)}
$$

Then, compute the autocorrelation function of $z(n)$

$$
\begin{aligned}
R(D) & =\sum_{n=1}^{M} z^{*}(n) z(n+D) \\
& =e^{j\left(2 \pi f_{c} D\right)} \sum_{l=0}^{L}\left|h_{n_{t} n_{r}}(l)\right|^{2} \sum_{n=1}^{N}|c(n)|^{2}|c(n+D)|^{2}
\end{aligned}
$$

and the estimated CFO is given by [25]

$$
\hat{f}_{c}=\frac{\arg (R(D))}{2 \pi D}
$$

Literatures [32] shows that the CFO variance of coherent autocorrelation algorithm under AWGN channel is

$$
\operatorname{var}\left(\hat{f}_{c}\right)=\frac{1}{(2 \pi D)^{2}}\left[\frac{1}{M^{2}} \frac{2}{(2 \mathrm{SNR})}+\frac{1}{M} \frac{2}{(2 \mathrm{SNR})^{2}}\right]
$$

With the multipath channel interference, its variance could deteriorate even further.

The performance of coherent autocorrelation algorithm under low SNR situation is bad. To solve this problem, we propose a differential correlation algorithm.

\subsection{Differential correlation algorithm}

According to the cyclic shift-and-add property of $\mathrm{m}$ sequence, the differential operation of the locally generated $\mathrm{PN}$ sequence can be expressed as [33].

$$
\begin{aligned}
c^{K}(n) & =\operatorname{circshift}(c(n), K) \\
& =c(n) \cdot c^{D *}(n) \\
& =c(n) \cdot \operatorname{circshift}\left(c^{*}(n), D\right)
\end{aligned}
$$

where $D$ is the differential delay, circshift(.) denotes cyclic shift, and $K$ is an integer related to $D$. That is to say, the differential operation of the PN sequence is still a PN sequence which has great autocorrelation property [29]. Let

$$
r^{D}(n)=\sum_{l=0}^{L-1} h_{n_{t} n_{r}}(l) c^{D}(n-l) e^{j 2 \pi(n-D-l) f_{c}}
$$

Then, the differential operation of the received PN sequence can be expressed as

$$
\begin{aligned}
r^{K}(n) & =r(n) \cdot r^{D *}(n)=\sum_{l=0}^{L-1} h_{n_{t} n_{r}}(l) c(n-l) e^{j 2 \pi(n-l) f_{c}} \\
& \times\left(\sum_{i=0}^{L-1} h_{n_{t} n_{r}}(i) c^{D}(n-i) e^{j 2 \pi(n-D-i) f_{c}}\right)^{*} \\
& =\sum_{l=0}^{L-1} h_{n_{t} n_{r}}(l) \sum_{i=0}^{L-1} h_{n_{t} n_{r}}^{*}(i) c(n-l) c^{D}(n-i) e^{j 2 \pi D f_{c}} \\
& =\sum_{l=0}^{L-1}\left|h_{n_{t} n_{r}}(l)\right|^{2} c^{K}(n-l) e^{j 2 \pi D f_{c}}+\varepsilon_{3}
\end{aligned}
$$

where

$$
\varepsilon_{3}=\sum_{l=0}^{L-1} \sum_{i=0, i \neq l}^{L-1} h_{n_{t} n_{r}}(l) h_{n_{t} n_{r}}{ }^{*}(i) c(n-l) c^{D}(n-i) e^{j 2 \pi D f_{c}}
$$

It can be seen that after the differential operation, the phase rotation of the received PN sequence caused by CFO is changed into a fixed phase difference $e^{j 2 \pi D f_{c}}$. Furthermore, the cross-correlation between $r^{K}(n)$ and $c^{K}(n)$ can be expressed as

$$
R_{r c, D}(s)=\frac{1}{M} \sum_{l=0}^{L-1}\left|h_{n_{t} n_{r}}(l)\right|^{2} \sum_{n=0}^{M-1} c^{K}(n) c^{K *}(n+s-l) e^{j 2 \pi D f_{c}}
$$

When $r^{K}(n)$ aligns with $c^{K}(n), L$ correlation peaks will appear, that is

$$
R_{r c, D}(s)=\left|h_{n_{t} n_{r}}(s)\right|^{2} e^{j 2 \pi D f_{c}}+\Delta \varepsilon_{3} \quad, s \in[0, L-1]
$$

where $\Delta \varepsilon_{3}$ is the interference term related to $\varepsilon_{3}$. We here choose the highest correlation peak to estimate the CFO [34].

$$
\hat{f}_{c}=\left.\frac{\arg \left(R_{r c, D}(s)\right)}{2 \pi D}\right|_{s=s_{0}}
$$

where $\arg (\cdot)$ denotes the phase of a complex number measured in radians, $s_{0}$ represents the location of the 
highest correlation peak. It is noteworthy that, since $-\pi \leq \arg \left(R_{r c, D}(s)\right) \leq \pi,-1 /(2 D) \leq \hat{f}_{c} \leq 1 /(2 D)$.

The estimated $\hat{f}_{c}$ will be used to compensate for the CFO effect on $R_{r c}(s)$ in Eq. (11). Then, we have

$$
\begin{aligned}
R_{r c}(s) & =\frac{1}{M} \sum_{n=0}^{M-1} c(n) \cdot r(n+s) e^{-j 2 \pi(n+s) \hat{f}_{c}} \\
& =\frac{1}{M} \sum_{l=0}^{L-1} h_{n_{t} n_{r}}^{*}(l) \sum_{n=0}^{M-1} c(n) c^{*}(n+s-l) e^{j 2 \pi(n+s-l)\left(f_{c}-\hat{f}_{c}\right)} \\
& =\left\{\begin{array}{c}
h_{n_{t} n_{r}}^{*}(s) \frac{1}{M} \sum_{n=0}^{M-1}|c(n)|^{2} e^{j 2 \pi n \Delta f_{c}}+\Delta \varepsilon_{3}, s \in[0, L-1] \\
\Delta \varepsilon_{4}, \text { otherwise }
\end{array}\right.
\end{aligned}
$$

where $\Delta \varepsilon_{4}$ is the interference term related to

$$
\varepsilon_{4}=\sum_{l=0}^{L-1} \sum_{i=0}^{L-1} h_{n_{t} n_{r}}(l) h_{n_{t} n_{r}}{ }^{*}(i) c(n-l) c^{D}(n-i) e^{j 2 \pi D f_{c}}
$$

and $\Delta f_{c}=f_{c}-\hat{f}_{c}$. If $\hat{f}_{c}$ is estimated precisely, then $\Delta f_{c} \approx 0$. Equation (24) can be rewritten as

$$
R_{r c}(s)= \begin{cases}h_{n_{t} h_{r}}^{*}(s)+\Delta \varepsilon_{3}, s \in[0, L-1] \\ \Delta \varepsilon_{4}, \text { otherwise }\end{cases}
$$

Still, the location of those peaks that exceed the threshold $\xi=4 E\left[R_{r c}^{2}\right]$ is extracted as PCS information of $\mathrm{h}_{n_{t} n_{r}}$, which can be expressed as

$$
\mathrm{I}_{n_{t} n_{r}}=\left\{\mathrm{s},\left\|R_{r c}(s)\right\|_{2} \geq \xi\right\}_{s=0}^{L-1}
$$

\section{Improved CS reconstruction algorithm}

According to the MIMO-OFDM model in Section 2, here, we only consider the frequency domain signal received in the location of pilot at $n_{r}$ th antenna, which can be expressed as

$$
\mathrm{Y}_{p}^{n_{r}}=\sum_{n_{t}=1}^{N_{t}} \operatorname{diag}\left(\mathrm{X}_{p}^{n_{t}}\right) \mathrm{F}_{p} \mathrm{~h}_{n_{t} n_{r}}+\mathrm{n}_{n_{r}, p}
$$

where $\mathrm{F}_{p} \in \mathbb{C}^{N_{P} \times L}$ and $\mathrm{n}_{n_{r}, p} \in \mathbb{C}^{N_{p} \times 1}$ are the DFT matrix and noise vection corresponding to pilot location, respectively. Equation (28) can be further simplified as

$$
\mathrm{Y}_{p}^{n_{r}}=\mathrm{A}_{n_{r}} \mathrm{~h}_{n_{r}}+\mathrm{n}_{n_{r}, p}
$$

where

$$
\begin{array}{r}
\mathrm{A}_{n_{r}} \triangleq\left[\operatorname{diag}\left(\mathrm{X}_{p}^{1}\right) \mathrm{F}_{p}, \operatorname{diag}\left(\mathrm{X}_{p}^{2}\right) \mathrm{F}_{p}\right. \\
\left., \ldots, \operatorname{diag}\left(\mathrm{X}_{p}^{N_{t}}\right) \mathrm{F}_{p}\right] \in \mathbb{C}^{N_{p} \times N_{t} L}
\end{array}
$$

is the measurement matrix and $\mathrm{Y}_{p}^{n_{r}}$ is also called an observation vector [26]. $\mathrm{h}_{n_{r}} \triangleq\left[\mathrm{h}_{1 n_{r}}^{T}, \mathrm{~h}_{2 n_{r}}^{T}, \ldots \mathrm{h}_{N_{t} n_{r}}^{T}\right]^{T} \in \mathbb{C}^{N_{t} L \times 1}$ is the vector to be estimated. In a typical massive MIMO-OFDM system, $N_{p} \ll N_{t} L$, which means Eq. (29) is undetermined equations. Fortunately, we can use the CS recovery algorithm to get the solution of Eq. (29), i.e., to estimate $\mathrm{h}_{n_{r}}$.

Greedy iteration CS reconstruction schemes are proved to be feasible in OFDM system channel estimation. However, for most conventional greedy iteration CS reconstruction schemes, the channel sparsity level has to be transmitted to receive end as a priori information [15], which is unachievable in normal actual systems. While the sparsity adaptive greedy iteration CS reconstruction schemes suffer from low accuracy and high computational complexity [16]. Neither of those two kinds of CS reconstruction scheme can effectively catch the time-varying channel information.

In our scheme, the CS recovery algorithm does not need channel level as a priori information. Its iteration is based on the PCS information and controlled by an iteration threshold. For convenience of expression, the PCS information of the $n_{r}$ th receive antenna is summarized as

$$
\mathrm{I}_{n_{r}}=\sum_{n_{t}=1}^{N_{t}}\left(\mathrm{I}_{n_{t} n_{r}}+\left(n_{t}-1\right) L\right)
$$

In the following algorithm steps, $t$ is iteration time and the th column index, updated measurement matrix, updated index, estimated channel vector, and residual are denoted by $\Lambda_{t}, \mathrm{~A}_{t}, \lambda_{t}, \mathrm{r}_{t}$, and $\hat{\mathrm{h}}_{n_{r}, t}$, respectively. To improve recovery accuracy, we set $\varepsilon \approx$ $10^{-6}$ as the iteration threshold. Our CS-based massive MIMO-OFDM channel estimation in the $n_{r}$ th receive antenna can be summarized as follows:

\footnotetext{
Algorithm 1 The improved CS-based channel estimation

Input:

(1) the measurement matrix $A_{n_{r}}$ the observation vector $Y_{p}^{n_{r}}\left(1 \leq n_{r} \leq N_{t}\right)$

(2) the PCS information $I_{n_{r}}\left(1 \leq n_{r} \leq N_{t}\right)$

Initialization

$\Lambda_{0}=I_{n_{r}}, A_{0}=\left.A_{n_{r}}\right|_{\Lambda_{0}}$

$r_{0}=Y_{p}^{n_{r}}-A_{0}\left(A_{0}^{T} A_{0}\right)^{-1} A_{0}^{T} Y_{p}^{n_{r}}$,

$t=0, \varepsilon \approx 0$

While $\left\|r_{t}\right\|_{2}<\varepsilon$ do
} 


\section{Improved CS reconstruction algorithm (Continued)}

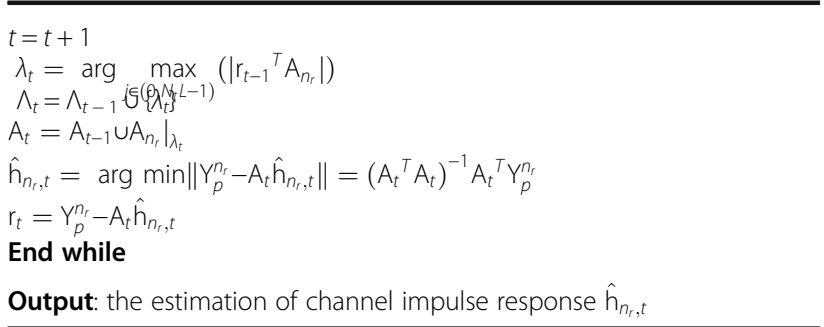

The channel of other receive antennas is also estimated according to the above methods. Unlike conventional greedy iteration CS reconstruction schemes such as OMP and CoSaMP, where the number of iterations is the value of channel sparsity level [20], the proposed scheme stops iteration only when the residual is close to $0\left(\left\|\mathrm{r}_{t}\right\|_{2}<\varepsilon\right)$. Therefore, the reconstruction accuracy can be guaranteed. Under the condition that the precise PCS information is obtained, the number of iterations in the proposed scheme is limited. That is to say, the computational complexity is controllable.

\section{Results and discussion}

In this section, we simulate the proposed CS-based channel estimation scheme under CFO environment. To evaluate the performance, conventional CS reconstruction schemes including OMP, CoSaMP, and SAMP are also simulated. For compare purpose, we consider the typical $8 \times 8$ and $16 \times 16$ massive MIMO-OFDM systems. It is noteworthy that random pilot insertion is exploited in the simulation as it can enhance restrain isometric property (RIP) of the measurement matrix [35]. The specific parameters of our system in the simulations are listed in Table 1.

In the following chapters, we will consider the typical six-taps ITU vehicular B (ITU-VB) and State Administration of Radio, Film, and Television 8 (SARFT-8) channel model as our simulation channel whose parameters are listed in Table 2.

\subsection{The CFO influence on PCS information acquisition}

Figure 3 plots the CFO influence on $R_{r c}$ in $16 \times 16$ MIMOOFDM system with $f_{\max }=20 \mathrm{HZ}$ and $\mathrm{SNR}=5 \mathrm{~dB}$. For comparison purpose, the actual CIRs of the used SARFT-8 and ITU-VB channel are marked in red. Normally (without CFO), as shown in Fig. $3 \mathrm{~b}$ and $\mathrm{d}$, the peaks of $R_{r c}$ will roughly overlap with the actual CIR and be obtained as PCS information. However, as shown in Fig. 3a and c, the peaks of $R_{r c}$ disappeared. That is because the amplitude of the peak is reduced quickly in Singer function way due to the effect of CFO. And the larger the CFO, the more serious the amplitude attenuation of the peaks. Moreover, the performance of the PCS information acquisition scheme is sensitive to the value of CFO. Even the 0.05 normalized CFO can completely destroy its performance.
Table 1 Parameters in the simulations

\begin{tabular}{ll}
\hline Length of the PN sequence $M$ & 255 \\
$\begin{array}{l}\text { Differential delay in differential } \\
\text { correlation algorithm } D\end{array}$ & 2 \\
System transmission speed & $20 \mathrm{Mbit} / \mathrm{s}$ \\
System bandwidth & $7.56 \mathrm{MHZ}$ \\
Center frequency & $634 \mathrm{MHZ}$ \\
Length of the OFDM block $N$ & 4096 \\
Number of pilots & 96 \\
Pilot insertion mode & Random \\
Baseband modulation method & $16 \mathrm{QAM}$ with 1/2 bitrate \\
& source coding \\
Channel model & 6 -tap ITU and 6-tap \\
& SARFT-8 vehicular-B \\
channel model
\end{tabular}

\subsection{The performance of CFO compensation}

To overcome the bad effect of CFO on PCS information acquisition, in this section, the coherent autocorrelation algorithm and the proposed differential correlation algorithm are implemented to estimate the actual CFO. For the purpose to evaluate the performance of CFO compensation, we adopt mean square error (MSE) as measurements, which can be expressed as

$$
\operatorname{MSE}\left(\hat{f}_{c}\right)=\frac{1}{N_{t} N_{r}} \sum_{i=1}^{N_{t}} \sum_{j=1}^{N_{r}}\left(\frac{\hat{f}_{c, i j}-f_{c, i j}}{f_{c, i j}}\right)^{2}
$$

where $f_{c, i j}$ is the CFO between the $i$ th transmit antenna and the $j$ th receive antenna.

Table 2 Multipath channel parameters for simulations

\begin{tabular}{llllll}
\hline Path index & \multicolumn{2}{l}{ ITU-VB } & & \multicolumn{2}{l}{ SARFT-8 } \\
\cline { 2 - 3 } & $\begin{array}{l}\text { Delay } \\
(\mu \mathrm{s})\end{array}$ & $\begin{array}{l}\text { Gain } \\
(\mathrm{dB})\end{array}$ & & $\begin{array}{l}\text { Delay } \\
(\mu \mathrm{s})\end{array}$ & $\begin{array}{l}\text { Gain } \\
(\mathrm{dB})\end{array}$ \\
\hline 1 & 0.00 & -2.5 & 0.00 & -18.0 \\
2 & 0.30 & 0.00 & 1.80 & 0.00 \\
3 & 8.90 & -12.8 & 1.95 & -20.0 \\
4 & 12.9 & -10.0 & 3.60 & -20.0 \\
5 & 17.1 & -25.2 & 7.50 & -10.0 \\
6 & 20.0 & -16.0 & 31.8, & 0.00 \\
\hline
\end{tabular}




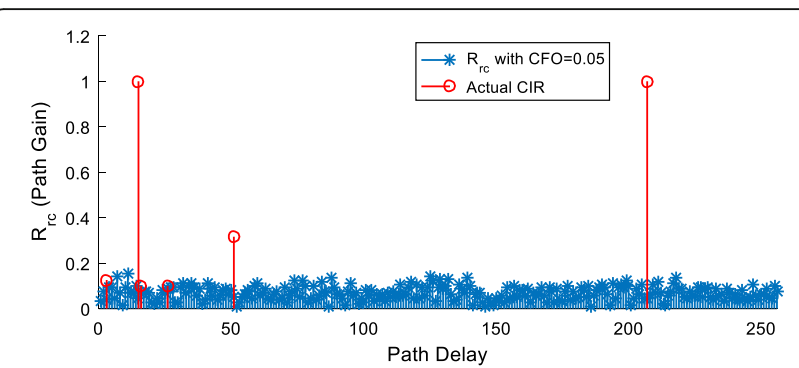

(a) $R_{r c}$ with CFO $f_{c}=0.05$ under SARFT-8 channel

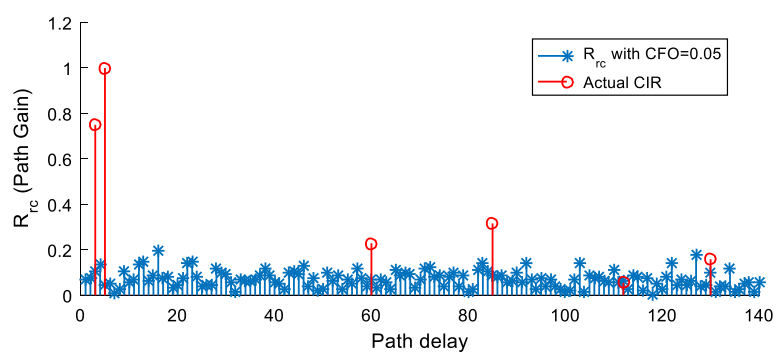

(c) $R_{r c}$ with CFO $f_{c}=0.05$ under ITU-VB channel

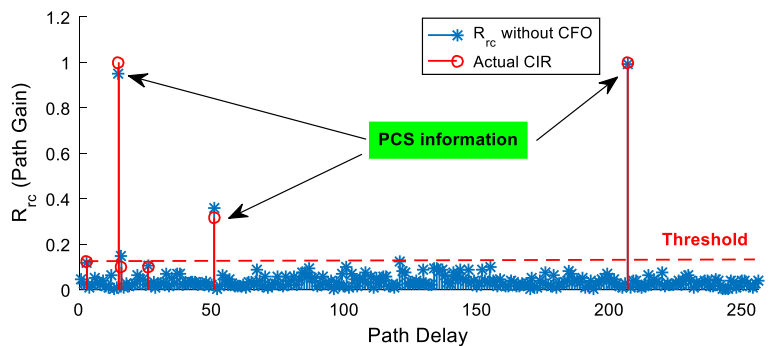

(b) $R_{r c}$ without CFO under SARFT-8 channel

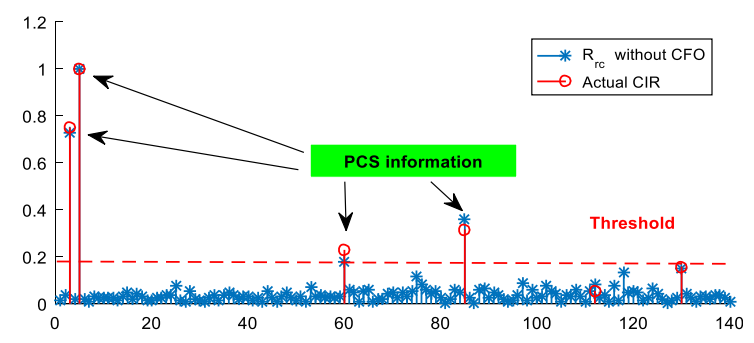

(d) $R_{r c}$ without CFO under ITU-VB channel

Fig. 3 The CFO influence on $R_{r C}$ (the cross-correlation between local PN sequence and received signal) in $16 \times 16 \mathrm{MIMO}-\mathrm{OFDM}$ system with $f_{\max }=20 \mathrm{HZ}$ and $\mathrm{SNR}=5 \mathrm{~dB}$

In Fig. 4, we show the MSE performance comparison of the above two CFO compensation schemes in $16 \times 16$ MIMO-OFDM system with $f_{\max }=20 \mathrm{HZ}$ under ITU-VB channel. As can be observed from Fig. 4a, when SNR= $5 \mathrm{~dB}$, the MSE of the coherent autocorrelation algorithm is higher than $10^{-1}$, which means the estimated CFO is unreliable. While the MSE of the proposed differential correlation algorithm is lower than $10^{-3}$, which shows that our scheme has better robustness to low SNR conditions. In Fig. 4b, we plot the MSE performance comparison of different CFO compensation schemes with $f_{c}=0.1$ and $f_{c}=$
0.05. As shown, when $\mathrm{SNR} \leq 20 \mathrm{~dB}$, the proposed algorithm outperforms the coherent autocorrelation algorithm in MSE performance. When SNR $>20 \mathrm{~dB}$, the performance of the two algorithms is similar.

Figure 5 presents the MSE of the estimated CFO in $16 \times 16$ MIMO-OFDM system with $f_{\max }=20 \mathrm{HZ}$. As shown, when SNR $\geq 15 \mathrm{~dB}$, for SARFT-8 channel, the MSE of $0.05,0.1$, and 0.2 normalized CFO are about $2 \times$ $10^{-4}, 8 \times 10^{-5}$, and $2 \times 10^{-5}$, respectively. For ITU-VB channel, the MSE of $0.05,0.1$, and 0.2 normalized CFO are about $4 \times 10^{-4}, 3 \times 10^{-4}$, and $8 \times 10^{-5}$, respectively.

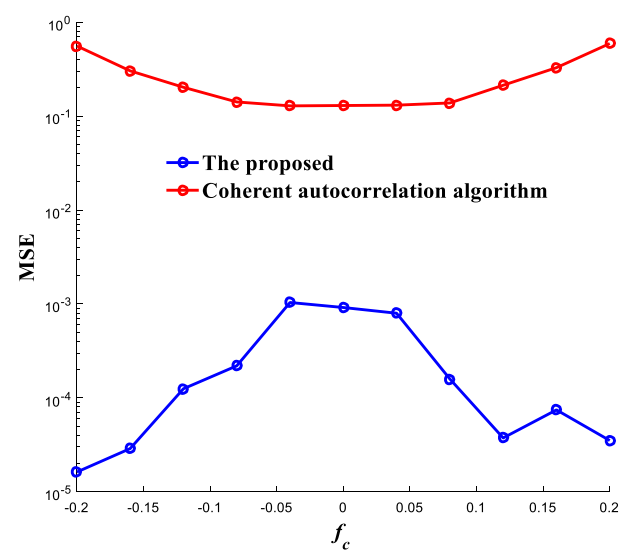

(a) MSE performance against $f_{c}$ with $S N R=5 d B$

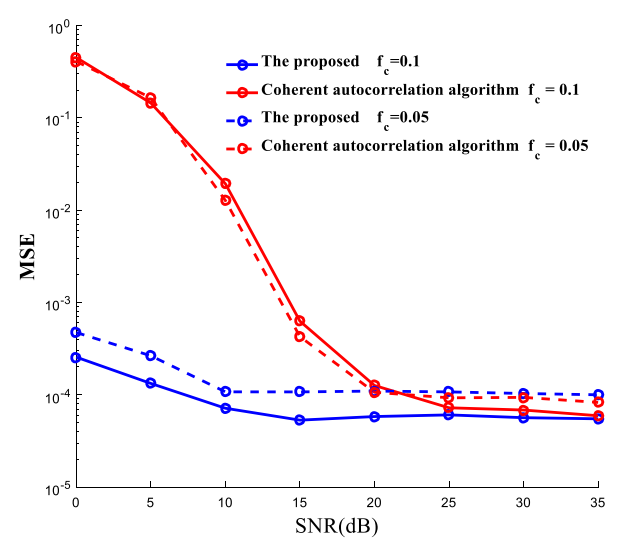

(b) MSE performance against SNR with $f_{c}=0.1$ and

$$
f_{c}=0.05
$$

Fig. 4 The performance comparison of two CFO compensation schemes in $16 \times 16$ MIMO-OFDM system 


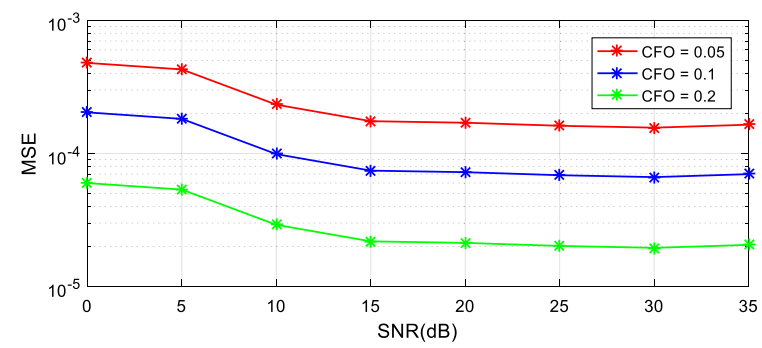

(a) MSE of the estimated CFO under SARFT-8 channel

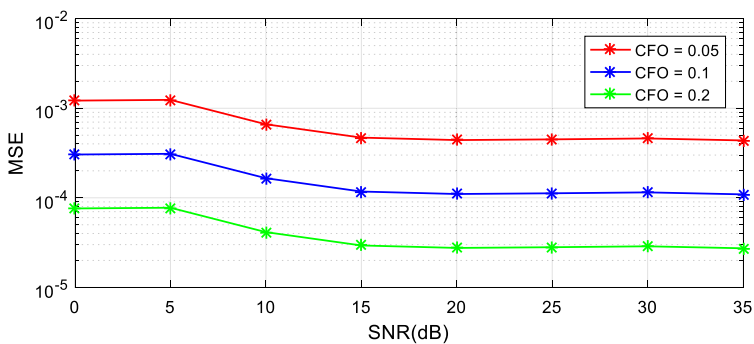

(b) MSE of the estimated CFO under ITU-VB channel

Fig. 5 The MSE performance of the proposed CFO compensation scheme in $16 \times 16 \mathrm{MIMO}-\mathrm{OFDM}$ system with $f_{\text {max }}=20 \mathrm{HZ}$

Even when SNR $=0 \mathrm{~dB}$, for both SARFT-8 and ITU-VB channel, the MSE of $0.05,0.1$, and 0.2 normalized CFO are still lower than $2 \times 10^{-3}, 3 \times 10^{-4}$, and $10^{-4}$, respectively. In other words, the performance of the robust differential correlation algorithm is limitly affected by noise. Furthermore, the MSE performance is affected by the channel. It can be seen that the MSE performance under SARFT-8 channel is better than that of ITU-VB channel.

Since the estimated CFO is accurate, the excellent CFO compensation of signals can be achieved. Figure 6 presents the cross-correlation $\left(R_{r c}\right)$ between local PN sequence and the CFO-removed receive signal in $16 \times 16$ MIMO-OFDM system with $f_{c}=0.05, f_{\max }=20 \mathrm{HZ}$, and $\mathrm{SNR}=5 \mathrm{~dB}$. Still, the actual CIRs of the used SARFT- 8 and ITU-VB channel are marked in red for comparison. Unlike Fig. 3a and c, after the CFO removal, the correlation peaks in Fig. 6 are evident and roughly overlap with the actual CIRs. By setting an appropriate threshold, the location of those peaks which are higher than the threshold is extracted as PCS information. Though the obtained PCS information is not accurate as the actual delay of CIRs (there are three missed peaks in PCS information acquisition in Fig. 6a, it can provide valuable prior information for the following CS-based channel estimation).

7.3 The performance of the proposed channel estimation Figure 7 presents the MSE of the proposed PCSinformation-based CS channel estimation scheme in $16 \times$
16 MIMO-OFDM system with $f_{c}=0.05$. As can be seen, both the MSE and BER performances are limitly influenced by Doppler shift. This is to say, the proposed PCSinformation-based CS channel estimation scheme has good robustness under time-varying channels. The major factor that impacts the accuracy of channel estimation is the SNR of channel.

Figure 8 shows the MSE and BER performance of the proposed PCS-information-based CS channel estimation scheme in $8 \times 8,16 \times 16$, and $32 \times 32 \mathrm{MIMO}-O F D M$ systems with $f_{c}=0.05$ and $f_{\max }=20 \mathrm{HZ}$. As can be observed, for both SARFT-8 and ITU-VB channel, with the increase of $N_{t}$ and $N_{n}$ both MSE and BER performances get worse.

Figure 9 compares the MSE and BER performances of different CS channel estimation schemes in $16 \times 16$ MIMO-OFDM system with $f_{c}=0.05$ and $f_{\max }=20 \mathrm{HZ}$. In our simulation, we assume that channel sparsity level is known to OMP and CoSaMP, and the step size of SAMP is set to 2. Among the four compared schemes, SAMP has the worst performance. More specifically, in Fig. 9a and c, at the target MSE of $10^{-3}$, other schemes have $5 \mathrm{~dB}$ SNR gain compared to SAMP. The reason is that SAMP does not use any prior information of channel, and its accuracy is greatly affected by the setting of parameters such as step size. As shown in Fig. 6, though the obtained PCS information is not accurate enough, the MSE and BER performance of the proposed scheme are superior to that of OMP and CoSaMP. Hence, the

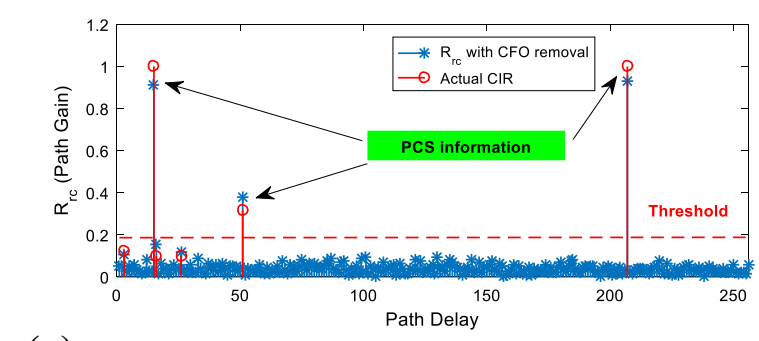

(a) PCS information acquisition with CFO removal under SARFT-8 channel

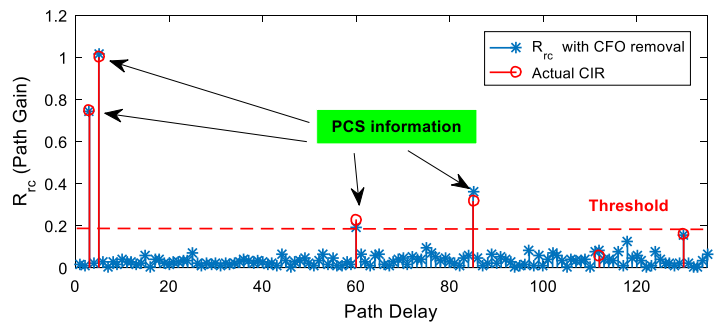

(b) PCS information acquisition with CFO removal under ITU-VB channel

Fig. 6 PCS information acquisition with CFO removal in $16 \times 16$ MIMO-OFDM system with $f_{\max }=20 \mathrm{HZ}, f_{C}=0.05$, and SNR $=5 \mathrm{~dB}$ 


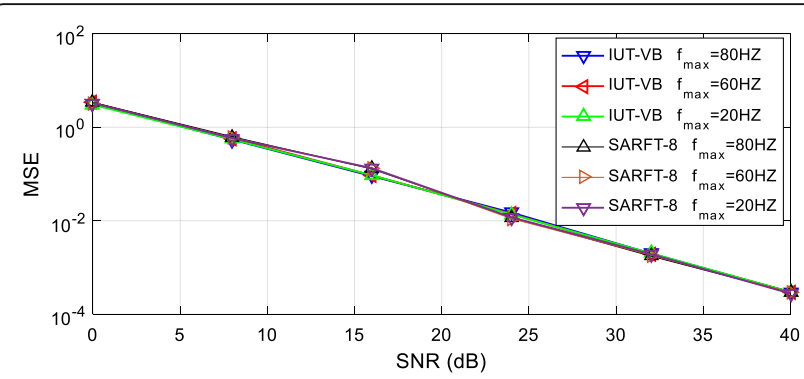

(a) MSE performance with $f_{c}=0.05$

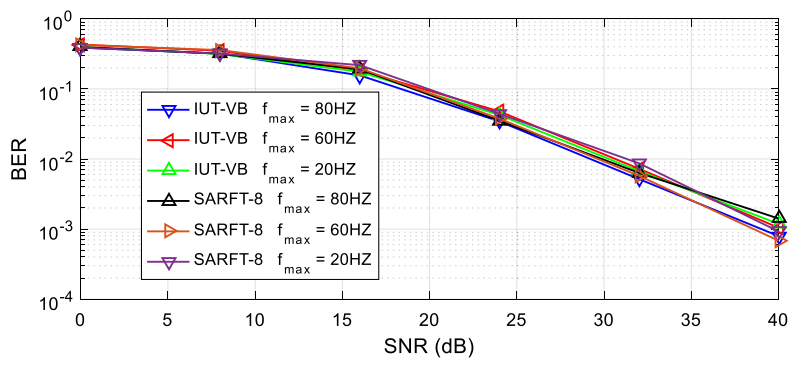

(b) BER performance with $f_{c}=0.05$

Fig. 7 The MSE and BER performance of the proposed PCS-information-based CS channel estimation scheme in $16 \times 16$ MIMO-OFDM system against $f_{\max }$

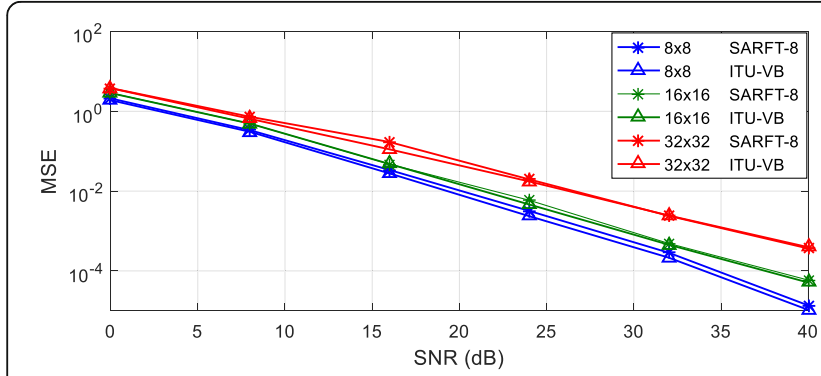

(a) MSE performance

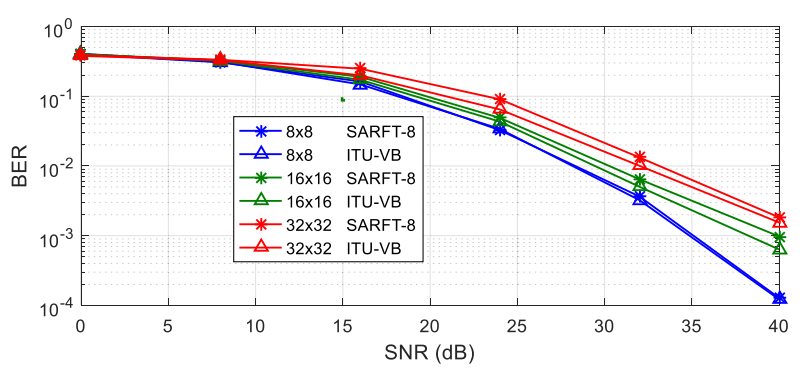

(b) BER performance

Fig. 8 The MSE and BER performance of the proposed PCS-information-based CS channel estimation scheme in $8 \times 8,16 \times 16$, and $32 \times 32$ MIMOOFDM systems with $f_{c}=0.05$ and $f_{\max }=20 \mathrm{HZ}$

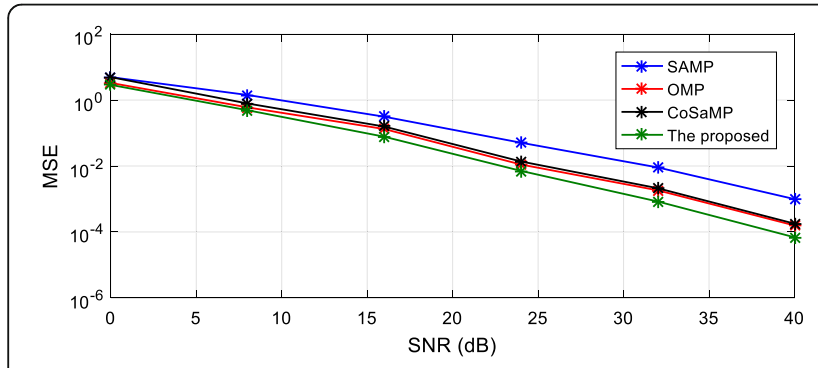

(a) MSE under SARFT-8 channel

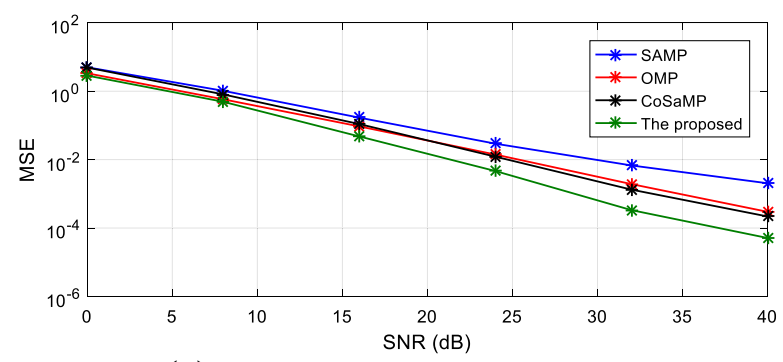

(c) MSE under ITU-VB channel

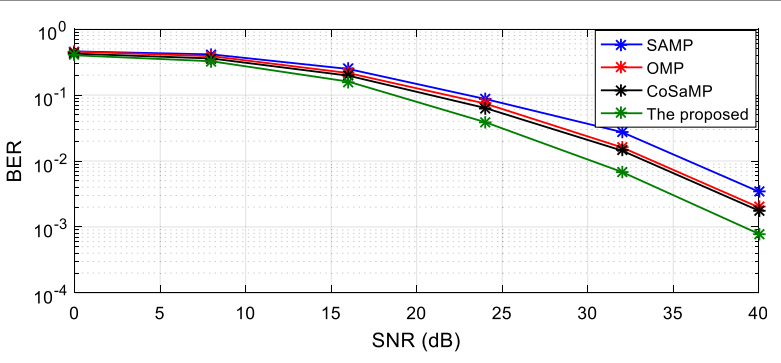

(b) BER under SARFT-8 channel

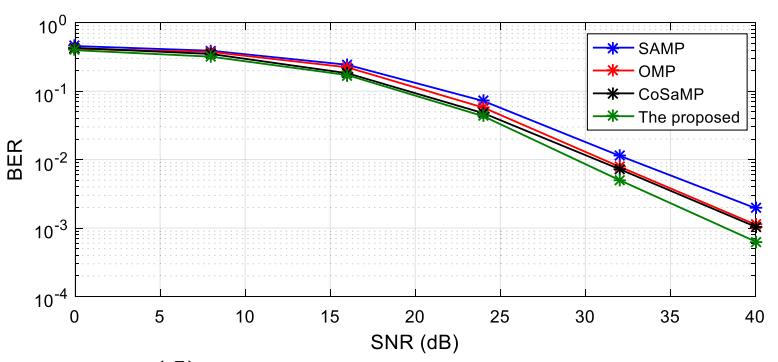

(d) BER under ITU-VB channel

Fig. 9 The performance comparison of different CS channel estimation schemes in $16 \times 16 \mathrm{MIMO}$-OFDM system with $f_{C}=0.05$ and $f_{\text {max }}=20 \mathrm{HZ}$ 
proposed scheme has great robustness under inaccurate PCS information situation.

Additionally, as can be seen, the performance result under ITU-VB channel is similar to that under SARFT-8 channel. The major difference is the performance gap between the proposed scheme and OMP/CoSaMP. As shown in Fig. 9c, the SNR gap between the proposed scheme and OMP/CoSaMP at MSE of $10^{-3}$ is about 5 $\mathrm{dB}$, which is higher than that under SARFT-8 channel. This is because the obtained PSC information is more accurate under ITU-VB channel (shown in Fig. 6).

As can be seen from the above results, the MSE and BER performances of the proposed scheme are better than other schemes. Moreover, compared with OMP and CoSaMP, there is no need to know the channel sparsity level as a priori information in the proposed scheme.

\section{Conclusions}

This paper discusses sparse massive MIMO-OFDM channel estimation based on compressed sensing over frequency offset environment. First, we propose a new OFDM frame structure, which the major benefit is low implementation complexity. Then, based on the proposed OFDM frame structure, the CFO is estimated by utilizing the proposed differential correlation algorithm, so its influence on channel estimation can be eliminated. Afterward, the PCS information of each channel is obtained. Based on the obtained PCS information, the CS reconstruction scheme is exploited to estimate the channel. The simulation result shows that the performance of the proposed scheme is superior to that of other mentioned schemes. Moreover, it does not need to know the channel sparsity level as a priori information; therefore, it is more practicable than other CS-based channel estimation schemes.

\begin{abstract}
Abbreviations
BER: Bit error rate; CFO: Carrier frequency offset; CIR: Channel impulse response; CoSaMP: Compressed sampling matching pursuit; CP: Cyclic prefix; CS: Compressed sensing; DFT: Discrete Fourier transform; DPN-OFDM: DualPN padded OFDM; IBI: Inter-block interference; ITU-VB: ITU vehicular B; LS: Least square; MIMO-OFDM: Multiple input multiple output orthogonal frequency division multiplexing; MMSE: Minimum mean square error; MSE: Mean square error; OFDM: Orthogonal frequency division multiplexing; OLA: Overlap and add; OMP: Orthogonal matching pursuit; PCS: Partial common support; PN: Pseudo noise; RIP: Restrain isometric property; SAMP: Sparsity adaptive matching pursuit; SARFT-8: State Administration of Radio, Film, and Television 8; SNR: Signal noise ratio; SP: Subspace pursuit
\end{abstract}

\section{Acknowledgements}

The authors would like to thank the NDSC (No.61401511 and No. U1736107) for their support and to anyone who supports this paper to be published.

\section{Authors' contributions}

$G L$ and $T L$ conceived of the algorithm and designed the experiments. GL, $X Z$, and $M X$ performed the experiments. GL and TL analyzed the results and drafted the manuscript. $G L, T L, M X$, and $X Z$ revised the manuscript. All authors read and approved the final manuscript.

\section{Authors' information}

Not applicable

\section{Funding}

This work was supported by the National Natural Science Foundation of China (No. 61401511) and the National Natural Science Foundation of China (No. U1736107)

\section{Availability of data and materials}

Data sharing is not applicable to this article as no datasets were generated or analyzed during the current study.

\section{Consent for publication}

Not applicable

\section{Competing interests}

The authors declare that they have no competing interests.

Received: 31 March 2019 Accepted: 21 June 2019

Published online: 08 July 2019

\section{References}

1. K. Ricardo, C.L. Paulo, G. Binghua, et al., Low-complexity and high-accuracy semi-blind joint channel and symbol estimation for massive MIMO-OFDM. Circ. Syst. Sig. Process 38(3), 1114-1136 (2018)

2. E. Konguvel, M. Kannan, A Survey on FFT/IFFT Processors for next generation telecommunication systems. J. Circ. Syst. Comput 27(03), 1830001-21 (2018)

3. T.S. Rappaport, S. Sun, R. Mayzus, H. Zhao, Y. Azar, K. Wang, G.N. Wong, J.K. Schulz, M. Samimi, F. Gutierrez, Millimeter wave mobile communications for 5 G cellular: it will work! IEEE Access 1, 335-349 (2013)

4. W.G. Song, J.T. Lim, Channel estimation and signal detection for MIMOOFDM with time varying channels. IEEE Commun. Lett 10(7), 540-542 (2006)

5. X. Lin, S. Wu, C. Jiang, et al., Estimation of broadband multiuser millimeterwave massive MIMO-OFDM channels by exploiting their sparse structure. IEEE Trans. Wirel. Commun 17(6), 3959-3973 (2018)

6. S.H. K, Y.H. You, J.H. Paik, et al., Frequency-offset synchronization and channel estimation for OFDM-based transmission. IEEE Commun. Lett 4(3), 95-97 (2002)

7. Henkel M, Schilling C, Schroer W, in Proc. of the IEEE Vehicular Technology Conference, Comparison of channel estimation methods for pilot aided OFDM systems, (2007), pp. 1435-1439

8. Khan A. M, Jeoti V, Zakariya M. A, in Proc. of the IEEE Symposium on Wireless Technology \& Applications, Improved pilot-based LS and MMSE channel estimation using DFT for DVB-T OFDM systems, (2013), pp. 120-124

9. Sutar M. B, Patil V. S, in Proc. of the International Conference on Electronics, Communication and Aerospace Technology, LS and MMSE estimation with different fading channels for OFDM system, (2017), pp. 740-745

10. H. Ye, G.Y. Li, B.H.F. Juang, Power of deep learning for channel estimation and signal detection in OFDM systems. IEEE Wirel. Commun. Lett 7(1), 114117 (2017)

11. F.G. Garcia, S. Zazo, J.M. Parez-Borrallo, Pilot patterns for channel estimation in OFDM. Electron. Lett 36(12), 1049-1050 (2000)

12. H. AL-Salihi, M. Nakhai, T. Le, Enhanced sparse Bayesian learning-based channel estimation for massive MIMO-OFDM systems. Eur. Conf. Netw. Commun. 1-5 (2017). https://doi.org/10.1109/EuCNC.2017.7980703

13. W.U. Bajwa, J. Haupt, A.M. Sayeed, et al., Compressed channel sensing: a new approach to estimating sparse multipath channels. Proc. IEEE. 98(6), 1058-1076 (2010)

14. D.J. Reinoso Chisaguano, Y. Hou, T. Higashino, et al., Low complexity channel estimation and detection for MIMO-OFDM receiver with ESPAR antenna. IEEE Trans. Veh. Technol 65(10), 8297-8308 (2016)

15. Taubock G, Hlawatsch F. in Proc. of the IEEE International Conference on Acoustics, Speech and Signal Processing, A compressed sensing technique for OFDM channel estimation in mobile environments: exploiting channel sparsity for reducing pilots, (2008), pp. 2885-2888

16. Jayanthi P. N, Ravishankar S, in Proc. of the IEEE International Conference on Recent Trends in Electronics, Sparse channel estimation for MIMO-OFDM systems using compressed sensing, (2017), pp. 1060-1064 
17. Yu Z, in Proc. of the $33^{\text {rd }}$ Chinese Control Conference, Variable step-size compressed sensing-based sparsity adaptive matching pursuit algorithm for speech reconstruction, (2014), pp. 7344-7349

18. H. Wu, S. Wang, Adaptive sparsity matching pursuit algorithm for sparse reconstruction. IEEE Signal Proc. Lett 19(8), 471-474 (2012)

19. Do T. T, Gan L, Nguyen N et al, in Proc. of the 42nd Asilomar Conference on Signals, Systems and Computers, Sparsity adaptive matching pursuit algorithm for practical compressed sensing, (2008), pp. 581-587

20. Bi X, Chen X. D, Zhang Y et al, in Proc. of the IEEE International Conference on Signal Processing, Variable step size stagewise adaptive matching pursuit algorithm for image compressed sensing, (2013)

21. W. Ding, F. Yang, W. Dai, et al., Time-frequency joint sparse channel estimation for MIMO-OFDM systems. IEEE Commun. Lett 19(1), 58-61 (2015)

22. J. Fu, J. Wang, J. Song, et al., A simplified equalization method for dual PNsequence padding TDS-OFDM systems. IEEE Trans. Broadcast 54(4), 825-830 (2008)

23. W. Ding, F. Yang, C. Pan, et al., Compressive sensing based channel estimation for OFDM systems under long delay channels. IEEE Trans. Broadcast 60(2), 313-321 (2014)

24. Q. Jing, M. Cheng, Y. Lu, et al., Pseudo-noise preamble based joint frame and frequency synchronization algorithm in OFDM communication systems, J. Syst. Eng. Electron 25(1), 1-9 (2014)

25. J. Wu, Y. Chen, $X$. Zeng, et al., Robust timing and frequency synchronization scheme for DTMB system. IEEE Trans. Consum. Electron. 53(4), 1348-1352 (2007)

26. Sheu CR, Huang CC A differential sliding correlation scheme for symbol timing detection in time domain synchronous OFDM systems. In Proceedings of the IEEE 69th Vehicular Technology Conference, Barcelona, Spain, 26-29 April 2009

27. Z. Li, X.G. Xia, An Alamouti coded OFDM transmission for cooperative systems robust to both timing errors and frequency offsets. IEEE Trans. Wirel. Commun 7(5), 1839-1844 (2008)

28. Prasad R, Murthy C R, Rao B D, Joint approximately sparse channel estimation and data detection in OFDM systems using sparse Bayesian learning[M]. IEEE Press, (2014), pp. 3591-3603

29. X. Ma, F. Yang, S. Liu, et al., Sparse channel estimation for MIMO-OFDM systems in high-mobility situations. IEEE Trans. Veh. Technol 67(7), 61136124 (2018)

30. L. Liang, J. Wang, J. Song, An improved spectrum sensing method for DTMB system based on PN autocorrelation. IEICE Trans. Commun 96(6), 1559-1565 (2013)

31. H.Y. Liu, Y.C. Yin, Efficient four-quadrant carrier frequency offset tracking for OFDM. J. Chin. Inst. Eng 40(6), 1-4 (2017)

32. M. Rezki, I. Kale, L. Sabel, Reduced complexity feedforward frequency offset estimator for AWGN communication channels. Int. Conf. Digit. Signal Proc 2 1313-1316 (2002)

33. F. Zeng, L. Ge, Proc. of the IEEE Information Theory Workshop, An algorithm for cycle and add property of m-sequence (2003), pp. 119-122

34. Wei J, Liu Y, in Proc. of the Second International Conference on Networks Security, Wireless Communications and Trusted Computing, Carrier frequency offset estimation using PN sequence iteration in OFDM systems, (2010), pp. 405-409

35. X. He, R. Song, W.P. Zhu, Pilot allocation for distributed compressed sensing based sparse channel estimation in MIMO-OFDM systems. IEEE Trans. Veh. Technol 65(5), 2990-3004 (2015)

\section{Publisher's Note}

Springer Nature remains neutral with regard to jurisdictional claims in published maps and institutional affiliations.

\section{Submit your manuscript to a SpringerOpen ${ }^{\circ}$ journal and benefit from:}

- Convenient online submission

- Rigorous peer review

- Open access: articles freely available online

- High visibility within the field

- Retaining the copyright to your article

Submit your next manuscript at $\boldsymbol{\nabla}$ springeropen.com 